\title{
Quality of Nursing Home: Spatial Patterns in Southwestern United States
}

\author{
S. Mantravadi \\ Health Economist \\ Kai Zhang \\ The University of Texas Health Science Center at Houston
}

Information systems, as well as applications of geographic information software and large databases, can draw light to issues apparent in the health care business. The purpose of this study was to explore the geographic trends in nursing home quality in Southwestern states, using the Nursing Home Compare dataset. Using Geographic Information Systems to evaluate nursing homes addresses quality of care in the context of payer expansion and the geographic dispersion of beneficiaries.

Keywords: Geographic Information System, Nursing Homes, Healthcare Quality

\section{INTRODUCTION}

There has been an influx of the baby boomer population into the healthcare industry; many of these individuals are eligible for or are currently receiving nursing home care. According to the National Nursing Home Survey, approximately 1.3-1.6 million elderly patients receive care from nursing homes (Castle \& Ferguson, 2010; Kaiser Family Foundation, 2015). Nursing Home residents often face low quality of care, and this has been a critical area of research (Castle \& Ferguson, 2010). Average quality of care for nursing home residents has consistently remained low (Werner \& Konetzka, 2010). More than $1 / 3^{\text {rd }}$ of nursing homes certified by Medicare or Medicaid have quality ratings of 1 or 2 stars, out of 5 stars, (Kaiser Family Foundation, 2015). Nursing home care is dominated by public insurance (50\% Medicaid, $16 \%$ Medicare), so the government and policy makers are invested/interested in ensuring that the public's money is well spent towards nursing home care (Kaiser Family Foundation, 2007; Werner \& Konetzka, 2010; Castle \& Ferguson, 2010). There are various definitions of nursing home quality; for example, in the nursing home compare data, there are 181 indicators to be considered (Castle \& Ferguson, 2010; CMS, 2016). Quality indicators are usually defined by measures of structure, process, and outcome (Castle \& Ferguson, 2010). Structural measures of quality include organizational structure and characteristics, while process measures concern what actually is done/occurs during the care process (Castle \& Ferguson, 2010). Finally, outcome measures evaluate health outcome (Castle \& Ferguson, 2010). Quality of life as a global measure of care has also been emphasized (Werner \& Konetzka, 2010). Nursing Home Compare data is publicly available, and provides standardized quality of care information (Castle \& Ferguson, 2010).

Several provisions of the Omnibus Budget Reconciliation Act of 1987 (Nursing Home Reform Act of 1987) made progress in the field of nursing home quality. This policy led to the implantation of 
Medicaid-Medicare certifications (15 months), inspections, and a comprehensive regulatory system, known as the Minimum Data Set (Werner \& Konetzka, 2010; Kaiser Family Foundation, 2007). The incorporation of the minimum data set (MDS), provides detailed information about resident quality of life, for quality improvement purposes (Kaiser Family Foundation, 2007). These regulations have posed minimum standards for nursing home quality (Werner \& Konetzka, 2010). Progress has been made regarding quality of care, such as a reduction in use of restraints, catheterization, functional decline, facility deficiencies, and hospitalizations (Werner \& Konetzka, 2010; Kaiser Family Foundation, 2007). However, these regulations alone are not the panacea/cure all for low quality of care; issues with quality still exist despite regulations and market reforms (Werner \& Konetzka, 2010).

The findings of low-quality ratings within nursing homes needs to be considered in the health care system organizational context, and foundations of quality measurement (Donabedian). Donabedian's model of Health Care Quality provides a theoretical foundation for evaluating quality in nursing homes. This model for quality can be aligned with several process improvement steps, and control of special cause variation; this study aims to evaluate geographical implications of the health care quality model. Many Structural, Process, and Outcomes based factors can be considered as quality of care measures in nursing homes, including staffing levels (number of nurses/staffs involved in care), ownership status, and deficiencies. Structural measures consider the health care organizational and system context, while Process measures provide insight into the health care delivery component involved in quality care. Finally, outcome measures consider the end all effect of healthcare on the patient population. These 3 measures in the Donabedian model will be considered as layers in this study.

The health care industry is dominated by nonprofit ownership, due to economic efficiency in the healthcare market. The failure of Contract theory (Hansmann) in the Healthcare market due to asymmetry of information, results in patients being more trusting of nonprofits (Folland, Goodman, \& Stano, 2013). Nonprofit ownership is also more of; this is an aspect that will be evaluated in the analyses. Further, the trust of ownership status may be a confounding factor - structure - in the association between quality ratings and factors. In for-profit nursing homes, deficiencies as identified by health inspection are higher, while quality ratings and staffing levels are lower (Harrington, Olney, Carrillo, \& Kang, 2012; Kaiser Family Foundation, 2015). Geographically, trends in structural measures of health care quality also differ. Geographic trends in both process and outcome measures exist as well.

At the process level, staffing levels is a layer to be considered at the geographic level (Mantravadi \& Zhang, 2017). Inadequate staffing levels are associated with low quality of care, and staffing levels have been stable at low levels in nursing homes for the past couple years (Kaiser Family Foundation, 2007). Although many studies evaluating nursing home staffing and quality of care have issues with sample size, study design, and quality indicators, the few methodologically robust studies indicate that there is an association between staffing levels (number of nurses/staffs involved in care) and quality of care (Castle, 2008).

Nursing home health inspections often reveal deficiencies, an outcomes measure. Nursing Home Compare is the most comprehensive and reliable data source for nursing home quality of care indicators (CMS, 2016). Data incorporates quality ratings information about the health inspections (deficiencies and penalties), staffing, and quality measures (CMS, 2016). Majority of the data contained in public reporting, such as Nursing Home Compare, evaluates a specific arena of quality of care including pressure sores, infections, pain, or unexplained weight loss (Werner \& Konetzka, 2010).

This study explored the geographic trends in nursing home quality in Southwestern states, using the Nursing Home Compare dataset. Geographic information systems was used to evaluate nursing home addresses for quality of care in the context of payer expansion and geographic dispersion of beneficiaries. The purpose for of this study was to explore the impact of ownership, nursing home health inspection deficiencies, and staffing levels on nursing home quality, as well as geographic trends in nursing home quality in the Southwestern states. Thus, in this study, nursing home ownership, staffing, and deficiencies will be incorporated as layers (for Structure, Process, and Outcomes measures of quality) into ArcGIS software, a geographic information systems (GIS) mapping software. The data used for this paper incorporates information from the Centers for Medicare and Medicaid Services Minimum Data Set 
(MDS) and Medicare/Medicaid inspections. Using geographic information systems to evaluate quality of care in nursing homes is necessary, as it is challenging to ensure optimal quality of care with the expansion of payers and changes in resident demographics due to the geographic dispersion of beneficiaries (Kaiser Family Foundation, 2007).

\section{METHODS}

The ArcGIS was used for mapping and spatial analysis of nursing home quality of care data. The usage of geographic information systems allows researchers to incorporate information technology when evaluating health in terms of the broader environment. Health and healthcare quality depend on location; and using geographic information systems aids in evaluating spatial autocorrelation in nursing home quality. In other words, use of such an information technology tool can explore if quality of care in nursing home "neighbors" are more related than nursing homes spaced farther apart. As per Tobler's First Law of Geography (spatial autocorrelation), "everything is related, but near things are more related than distant things" (Esr, n.d.a).

The data is from the Nursing Home Compare dataset, which incorporates data from the Centers for Medicare and Medicaid Services' (CMS) Health Inspections and the Minimum Data Set. The CMS Minimum Data Set is mandatory for Medicare-and-Medicaid certification (CMS reimbursement), and includes information about aspects of resident health (CMS, 2016). This data source contains data from every nursing home that is certified by Medicare and Medicaid (reimbursed by the centers of Medicare and Medicaid Services), and provides information about quality of care from Q32014 to Q12015. However, data regarding nursing homes that are not Medicare or Medicaid certified are not included (CMS, 2016).

The data was filtered to include only Medicare and Medicaid certified nursing homes in the South West region of the United States. According to the United States Census Bureau designated regions and divisions, the West South-Central Southwest division/region is defined as the states of Arkansas, Louisiana, Oklahoma, and Texas (U.S. Census, 2016, 2015). The data includes information on nursing home providers' addresses and zip codes. Using the Texas A\&M University (TAMU) Department of Geography, TAMU GeoServices, addresses were geocoded into ArcGIS. The TAMU GeoServices is an Environmental Sciences Research Institute (Esri n.d.a.) Development Center (EDC). For this study, layers incorporated were provider information, quality of care, deficiencies, penalties, ownership, and state averages of quality measures.

The Nursing Home Compare data was downloaded as several csv files, one file for each layer. Each file was imported into ArcGIS, using the add ASCII data feature. The Geoprocessing Join function was used to join the nursing home quality of care, deficiencies, penalties, ownership, and state averages files with the geocoded provider information file. Maps of nursing homes and an overall map of the nursing home providers in the southwest region of the United States were generated. The primary measure of nursing home quality was the quality rating, from the MDS and health inspection surveys in the Nursing Home Compare data; the staffing rating and health inspection deficiency ratings were also considered in analyses.

Both Statistical and spatial analytic techniques were used to evaluate correlations within features in the data, in addition to descriptive spatial views of health care services.

For spatial analysis of nursing home providers, several methods were used. Considering the geographic distribution of the data, the central feature of high-quality nursing homes in each state was identified. The central feature considers the most centrally located, in this case, nursing home; the central feature was used in order to ensure that the center that was identified occurred at a feature (or in this case, at a nursing home location). In this analysis, the central feature indicates the provider that has smallest distance between all other high-quality nursing homes.

Currently, there is a dearth of literature on the average distance between nursing homes. Information is also available on the average distance between a nursing home and hospice facilities, or the distance between a non-profit and for-profit nursing homes (Zheng, Mukamel, Friedman, Caprio, \& Temkin- 
Greener, 2015). Thus, for spatial analysis, the average distance between a nonprofit and a for-profit nursing home was used $(3.673 \mathrm{~km})$ as a baseline for proximity analyses conducted in this study. Thus, the proximity buffers in this study evaluated whether, on average, the nearest nursing home of similar quality (high or low quality) was located within 3.673 kilometers. In order to identify nursing homes with high quality measures, attribute extraction was used. Within ArcMap, the select attribute by attributes option was used for quality measures greater than or equal to 3 (high quality). Then, point proximity buffers were created for nursing homes with high quality/staffing ratings, and for nursing home providers with lower ratings. In other words, proximity analyses conducted in this study analyzed if a high-quality nursing homes located within $3.673 \mathrm{~km}$ of another high-quality nursing home.

To evaluate the extent of spatial autocorrelation (akin to Pearson's correlation coefficient), cluster analysis was performed, to assess the cluster of high quality and staffed nursing homes and the cluster of low quality and low-staffed nursing homes. Since ratings are categorical variables (5-point scale, with 5 being the best), the Average Nearest Neighbor index (ANNI) method was used. The ANNI will evaluate clustering, dispersion, and random distribution. Thus, clustering of nursing homes indicates that similar rated nursing homes are clustered (High Quality-High quality). Therefore, the select by attribute function was be used within the high-quality nursing home layer to choose relevant nursing homes in each state (Arkansas, Louisiana, Oklahoma, and Texas). Currently, a cluster analysis for all 4 states was conducted; clustering within each state was analyzed. In addition, clustering of nursing homes by ownership will be evaluated, statewide.

From a statistical perspective, histograms were used to descriptively evaluate the data distributions of each outcome variable. The Chi square analyses were also conducted in order to evaluate if there is quality measures are correlated with each other. Fisher's exact test was performed to determine if there is an association/relationship between ownership and quality measures; the exact test was used since one or more of the cells had less than 5 observations.

\section{RESULTS}

There were a total of 2,304 nursing home providers included in the sample. The data consisted of points, as the locations of each nursing home provider. As expected, nursing homes were clustered in metropolitan areas and bigger cities, with higher populations. This reflects the demand/need for these healthcare services.

Several maps were created, illustrating the locations of all nursing home providers in the Southwestern states; an overview map is illustrated in Figure 1. 


\section{FIGURE 1 \\ MAP OF NURSING HOMES IN SOUTHWESTERN UNITED STATES}

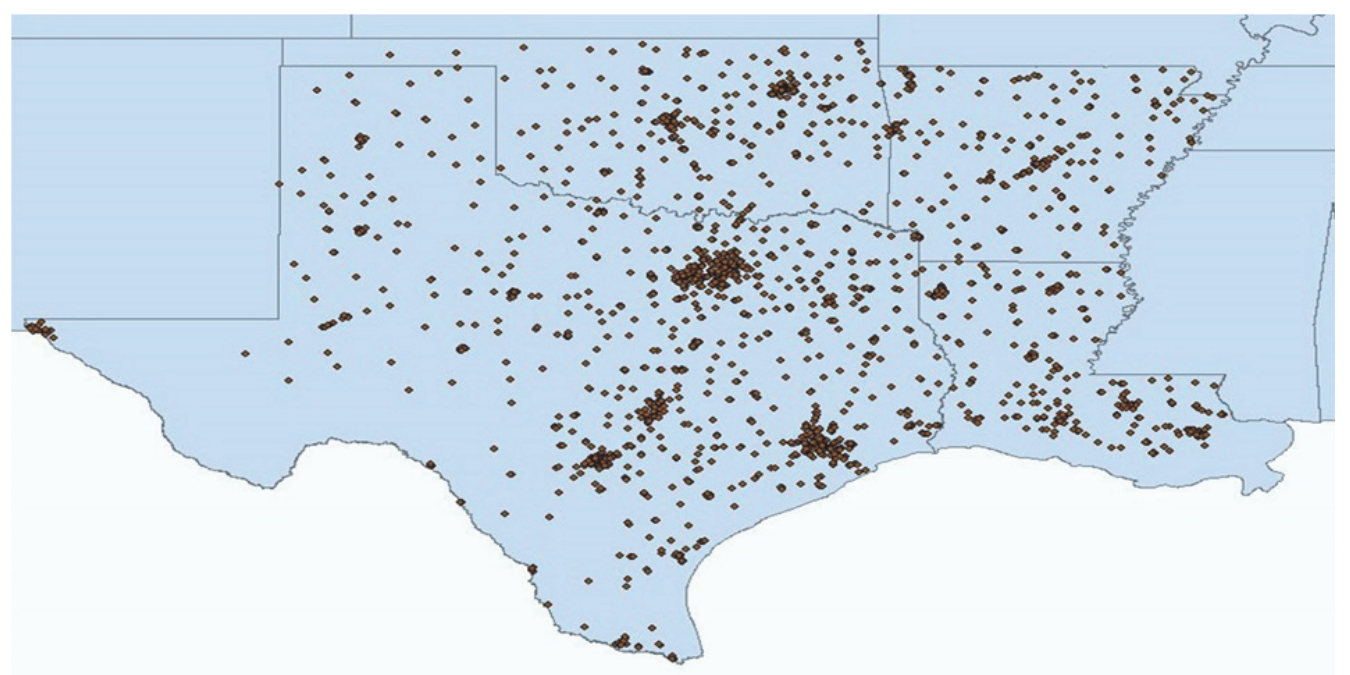

Each Point Represents A Nursing Home Facility

Figure 2 shows the distribution of quality ratings in nursing homes. Overall, from the map, it is evident that the previous literature on nursing home quality is confirmed. A majority of the sample of 2,034 nursing homes have low quality ratings, from 1-2 stars (as shown in red and yellow), with a few nursing homes rated as 3 (pale green). In metropolitan areas, such as Dallas, TX and New Orleans, LA, quality ratings are higher than in other rural areas in each of the states.

\section{FIGURE 2 \\ COLOR-CODED MAP: NURSING HOMES IN SOUTHWESTERN U.S.: QUALITY RATING}

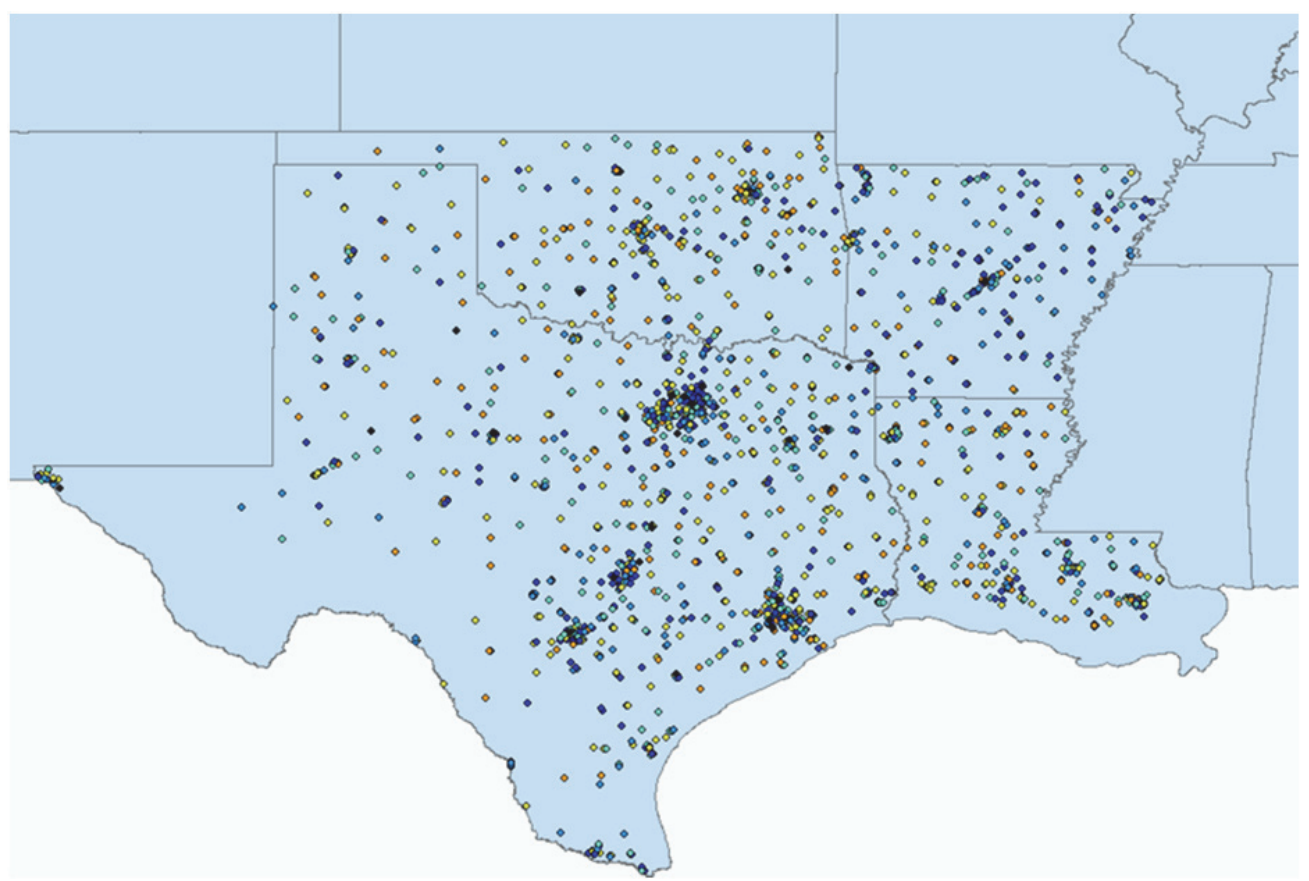

0 Stars: Red, 1 Star: Yellow, 2 Stars: Pale Green, 3-4 Stars: Light Blue, 5 Stars: Dark Blue Black Dots: Unavailable Data 
Similarly, a majority of the nursing home providers in the southwestern U.S. have low staffing ratings, from 1-2 stars, illustrating that nursing homes are understaffed, as in Figure 3. Contrary to quality ratings, low staffing ratings occur even in big cities/metropolitan areas.

The majority of nursing homes in the southwest have low health inspection ratings (higher deficiencies); this is evident within superimposed inspection data on location-based provider maps. In metropolitan areas, such as Dallas, TX, nursing homes with higher health inspection ratings are grouped together. Graphical Maps of ownership status of nursing home providers in Texas showed that a majority of nursing homes were for-profit. This is consistent with economic literature on nursing home ownership status. In the southwestern states, especially in major cities in Texas, for-profit nursing homes dominate. Very few nonprofit nursing homes were located in these southwestern states, and those non-profit homes were spread out throughout each state.

\section{COLOR-CODED MAP: NURSING HOMES IN SOUTHWESTERN U.S.: STAFFING RATING}

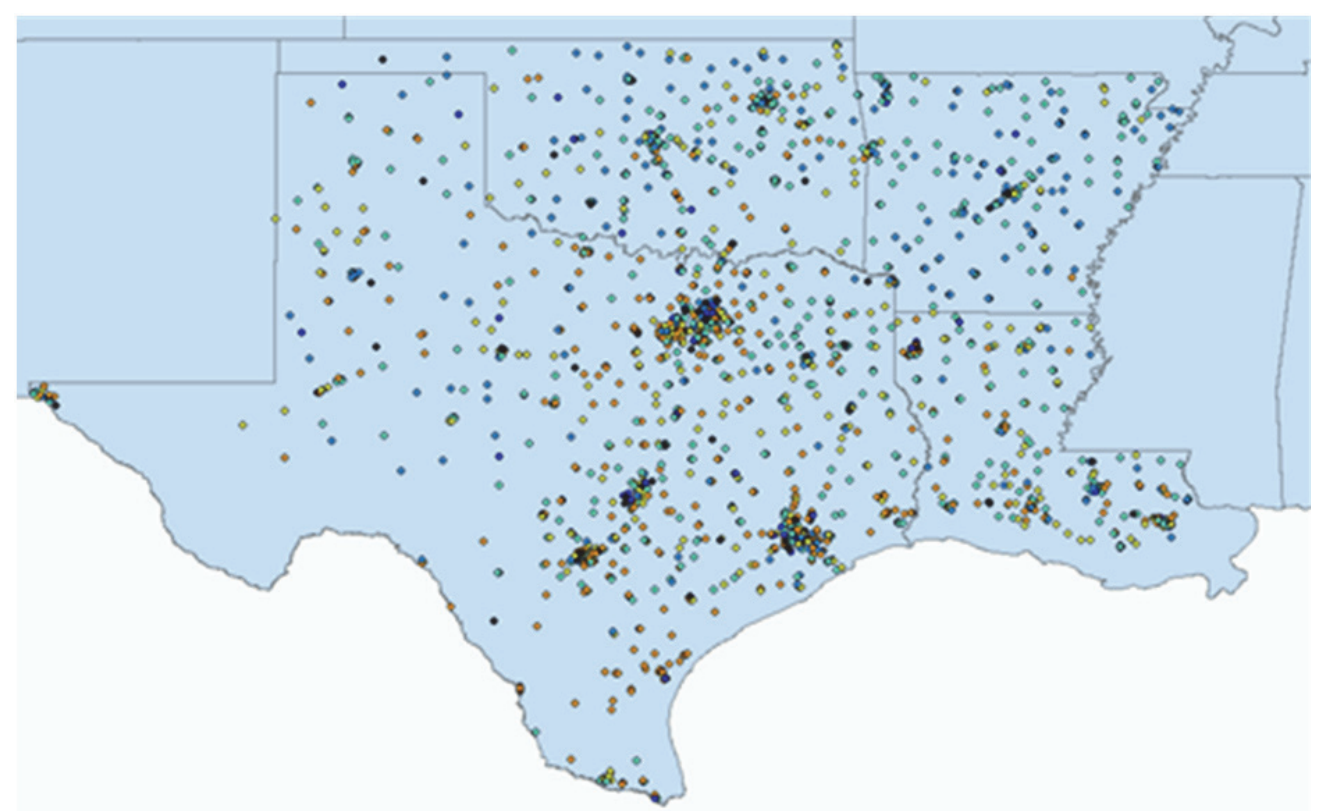

0 Star: Red, 1 star: Yellow, 2 Stars: Pale Green, 3 stars: Light Blue, 4-5 Stars: Dark Blue Black Dots: Unavailable Data

\section{Spatial Analysis}

The central features for high quality nursing homes in each of the states were calculated. In general, the central feature occurs in metropolitan areas; however, due other wide expanse of each of these southwestern states, the central feature is often located far away from other nursing homes. In Texas, this occurs near the metropolitan area of Dallas.

The results of the ANNI for high quality nursing homes indicated that there was statistically significant clustering, in each of the states in the southwest reagion. In Texas, the ANNI indicated positve, mild clustering among high quality nursing homes (ANNI $=0.41)$. The cluster analysis indicated that the ANNI was the highest for Oklahoma, and the lowest for Texas. This is expected, as Texas has a wider expanse of land compared to other southwestern states; nursing homes are clustered in metropolitan areas, and nursing homes are spread further out in rural areas of Texas. In Oklahoma, high quality nursing homes are randomly distribution throughout the state.

Except for Oklahoma, high quality nursing homes exhibit weak clustering in each state, as the ANNIs are closer to zero. In Oklahoma, high quality nursing homes are randomly distributed, as evidenced with 
an ANNI value of 0.81. Further research will aid in evaluating the spatial distance between nursing homes.

For high quality nursing homes, proximity buffers were also calculated. Results indicated that only in metropolitan areas did the national average distance between nursing homes; in rural areas, high quality nursing homes were spaced further apart. Considering Texas as an example, in rural areas, especially in west Texas, nursing homes are spaced farther apart. In Dallas, Houston, and near sections of San Antonio, the national average of $3.667 \mathrm{~km}$ distance between nursing homes seems more appropriate; here, the proximity buffers indicate that high quality nursing homes are located within $3.673 \mathrm{~km}$ of each other. However, the location of nursing homes is a function of the demand for health services, and affected by the population size. This is evident as in sparsely population regions of the southwest have nursing homes that are widely spread out. Additional proximity buffer maps considering the examples of Arkansas and Louisiana are in the appendix.

Figure 4 indicates a zoomed map of proximity buffers of high-quality nursing homes in Houston. In metropolitan areas such as Houston, TX, there was several high-quality rated nursing homes available within $3.673 \mathrm{~km}$ of a high-quality nursing homes. In figures 5 and 6 it is evident that using a proximity buffer of $3.673 \mathrm{~km}$ (national average) is not ideal. The above figures illustrate that in rural areas, high quality nursing homes are spaced farther apart, as spatial location of nursing homes becomes dependent on population size and demand for care.

\section{PROXIMITY BUFFER OF HIGH-QUALITY NURSING HOMES IN HOUSTON AREA, TX}

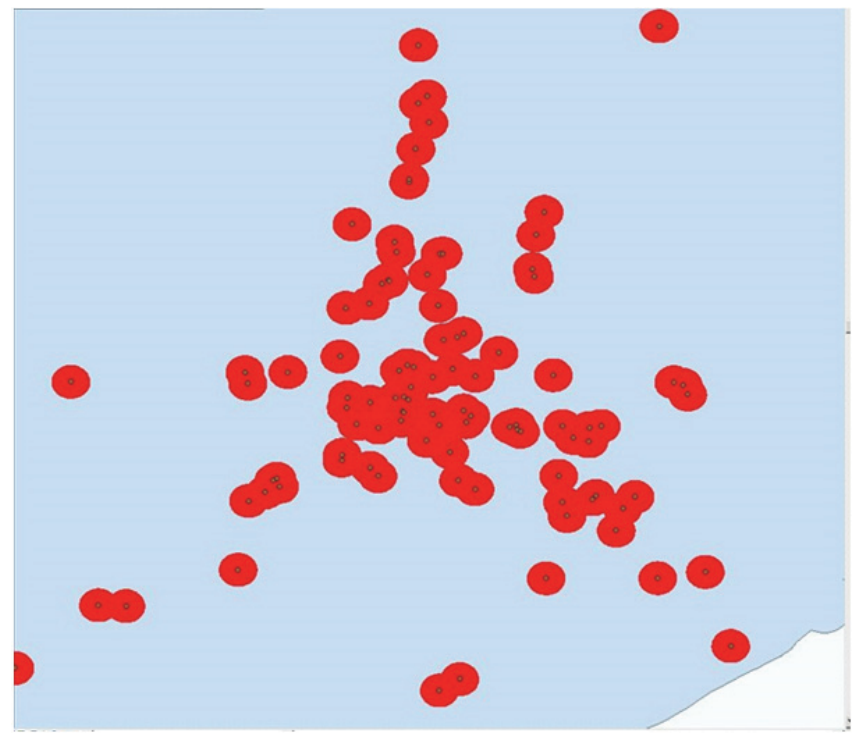

Bright Red Circles $=$ Proximity Buffer

Dark Maroon Points $=$ Nursing Home Locations 


\section{FIGURE 5}

\section{PROXIMITY BUFFER OF HIGH-QUALITY NURSING HOMES IN EAST TEXAS}

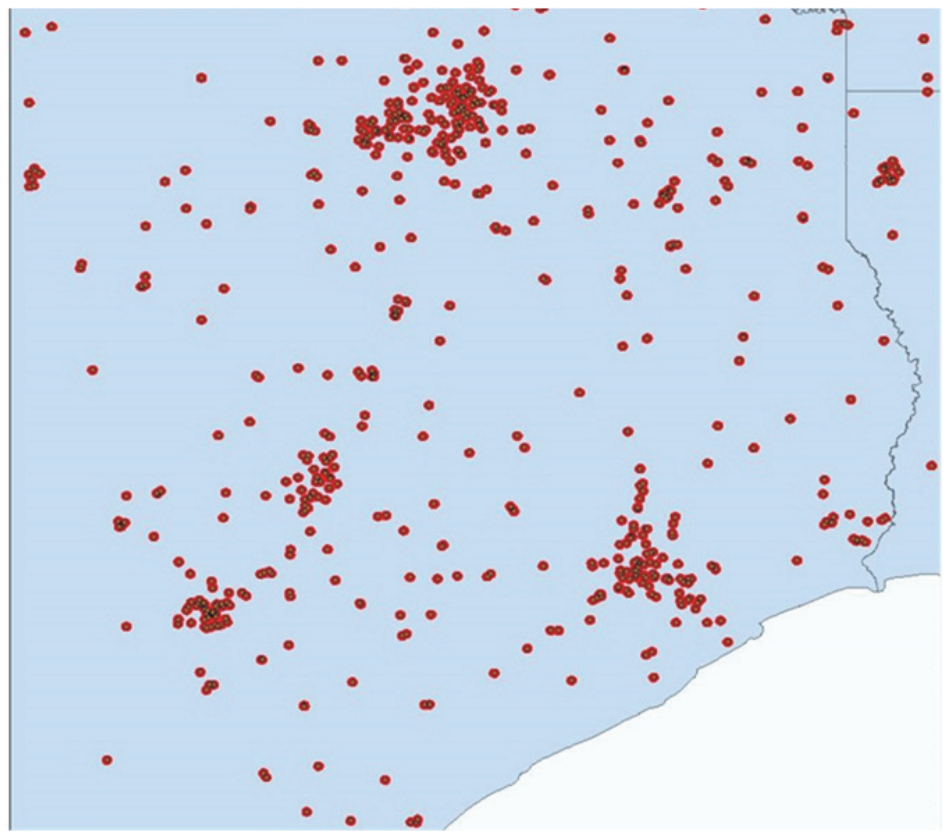

Bright Red Circles $=$ Proximity Buffer

Dark Maroon Points $=$ Nursing Home Locations

FIGURE 6

PROXIMITY BUFFER OF HIGH-QUALITY NURSING HOMES IN WEST TEXAS

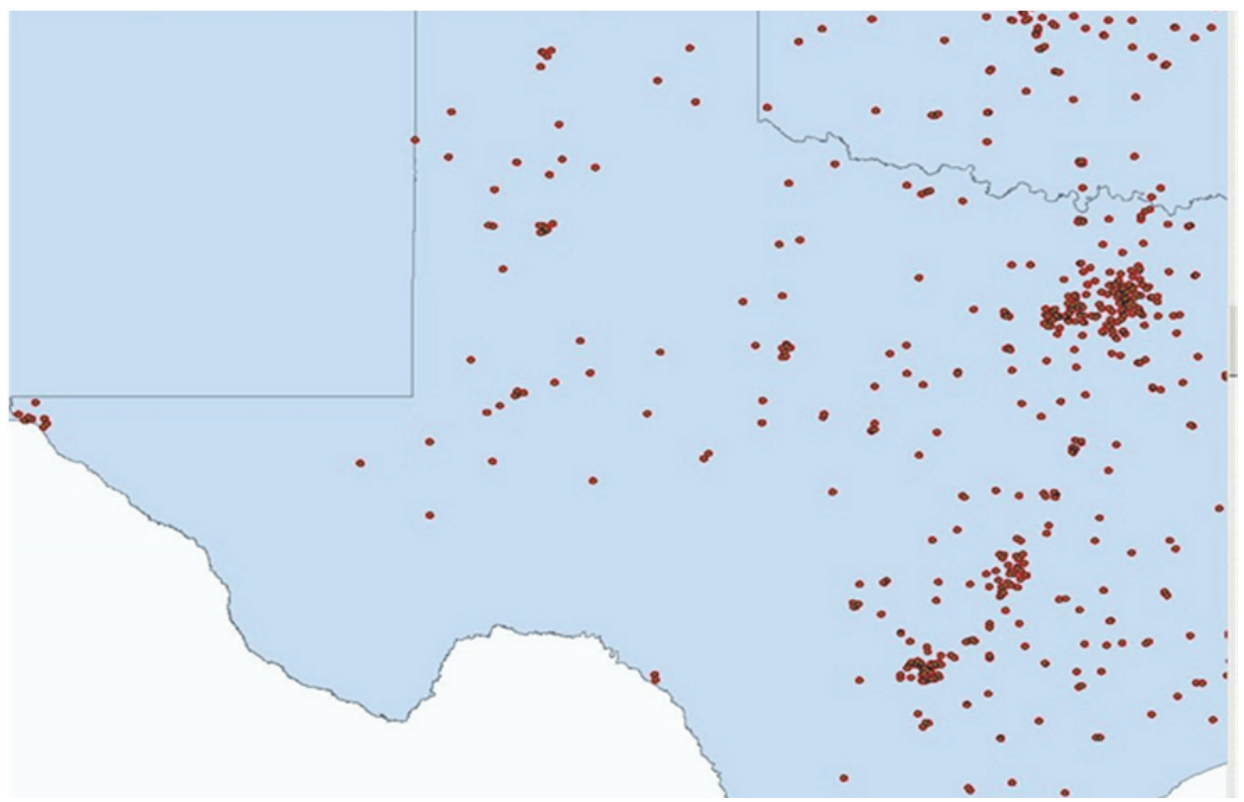

Bright Red Circles $=$ Proximity Buffer

Dark Maroon Points $=$ Nursing Home Locations

Considering the cluster analysis for staffing ratings, the ANNI for the staffing ratings ranged from 0.49 to 0.68 , indicating random distribution of high staffing ratings (akin to high quality rating) in each 
state. There is slight evidence of clustering, as the values of the ANNI are closer to zero than to 1. Thus, nursing homes with high staffing are weakly clustered; in general, providers who are adequate or high staffing are spatially located together.

The cluster analysis results are similar for the health inspection deficiencies and ownership status. All states indicated evidence of weak clustering by high health inspection ratings. Oklahoma tended towards a random distribution of nursing homes that passed the health inspection. As the ANNI tends to less than 1 , there is a trend of clustering by ownership and health inspection status in each state (Esri, n.d.b.).

\section{Statistical Analyses}

In order to verify the data distribution of quality measures, a histogram was used to evaluate statistical measures of central tendency for quality, staffing, and deficiency ratings. For the Health Inspection rating, values were distribution around 2 and 4 stars. The most of the nursing homes in the sample have a quality rating of 2 or 5 . The distribution of the staffing rating is moderate - a majority of the nursing homes have a staffing rating of 3 .

The Fisher's exact test, indicated there is an association between quality and ownership status, confirming the results from the previous literature (Grabowski, Feng, Hirth, Rahman, \& Mor, 2013). The chi square test underestimates, since $54 \%$ of the cells are less than 5 observations. Essentially, the relationship between quality rating and ownership status is statistically significant $(p<.0001)$. Thus, we reject to the null hypothesis. In other words, the variation in quality rating is likely due to ownership status.

Health inspection ratings and staffing ratings are correlated with ownership status. Table illustrates the Fisher's exact test results concerning ownership status and health inspection ratings. Similarly, Table 2 indicates that staffing rating varies by ownership status. Here, the variation in both staffing rating and health inspection ratings are due to ownership status.

The chi-square tests indicate that quality of care measures are strongly correlated with each other. Staffing is strongly related to health inspection ratings. Similarly, quality of care is correlated with staffing, and variation in health inspection ratings are associated with staffing levels. The variation in quality measures is not due to random chance.

TABLE 1

FISHER'S EXACT TEST: OWNERSHIP STATUS AND HEALTH INSPECTION RATING

\begin{tabular}{lrrrrrr}
\hline \multirow{2}{*}{ OWNERSHIP } & $\mathbf{9}$ Health Inspection Rating & \\
& $\mathbf{1}$ & $\mathbf{2}$ & $\mathbf{3}$ & $\mathbf{4}$ & $\mathbf{5}$ & Total \\
For-profit - & 258 & 301 & 290 & 310 & 118 & 1277 \\
Corporation & 12.82 & 14.95 & 14.41 & 15.4 & 5.86 & 63.44 \\
& 20.2 & 23.57 & 22.71 & 24.28 & 9.24 & \\
& 65.48 & 64.32 & 63.88 & 63.66 & 56.19 & \\
For-profit - & 28 & 26 & 30 & 18 & 7 & 109 \\
Individual & 1.39 & 1.29 & 1.49 & 0.89 & 0.35 & 5.41 \\
& 25.69 & 23.85 & 27.52 & 16.51 & 6.42 & \\
For-profit - & 7.11 & 5.56 & 6.61 & 3.7 & 3.33 & \\
Limited & 2 & 5 & 0 & 1 & 3 & 11 \\
Liability & 0.1 & 0.25 & 0 & 0.05 & 0.15 & 0.55 \\
& 18.18 & 45.45 & 0 & 9.09 & 27.27 & \\
For-profit - & 0.51 & 1.07 & 0 & 0.21 & 1.43 & \\
Partnership & 60 & 74 & 62 & 60 & 30 & 286 \\
& 2.98 & 3.68 & 3.08 & 2.98 & 1.49 & 14.21
\end{tabular}




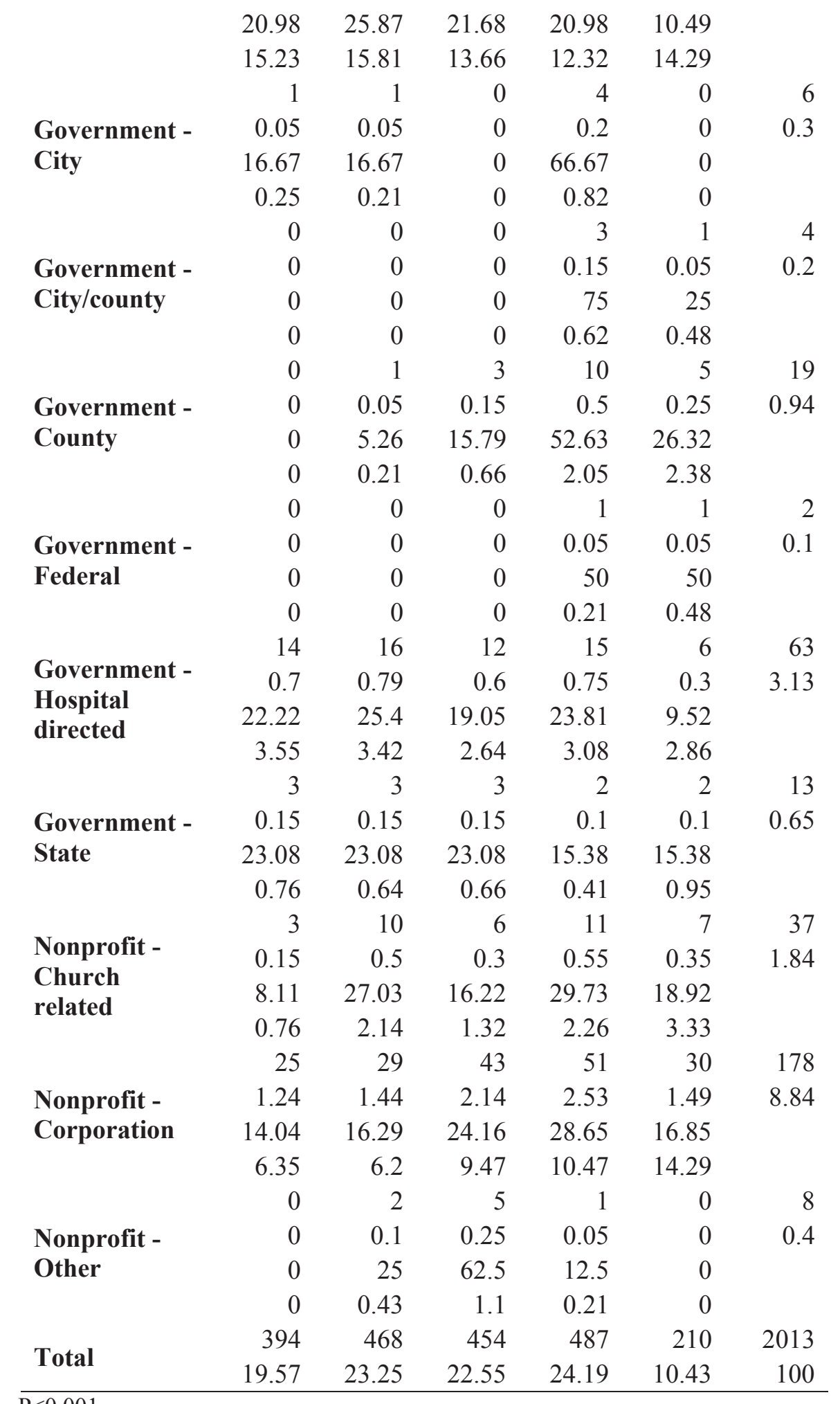

$\mathrm{P}<0.001$ 
TABLE 2

FISHER'S EXACT TEST: OWERNSHIP STATUS AND STAFFING RATING

\begin{tabular}{|c|c|c|c|c|c|c|}
\hline \multirow{3}{*}{ OWNERSHIP } & \multicolumn{6}{|c|}{ Staffing Rating } \\
\hline & 1 & 2 & 3 & 4 & 5 & Total \\
\hline & 361 & 259 & 417 & 191 & 30 & 1258 \\
\hline \multirow{4}{*}{$\begin{array}{l}\text { For-profit - } \\
\text { Corporation }\end{array}$} & 18.38 & 13.19 & 21.23 & 9.73 & 1.53 & 64.05 \\
\hline & 28.7 & 20.59 & 33.15 & 15.18 & 2.38 & \\
\hline & 66.98 & 65.4 & 65.57 & 59.87 & 40.54 & \\
\hline & 29 & 23 & 38 & 16 & 2 & 108 \\
\hline \multirow{4}{*}{$\begin{array}{l}\text { For-profit - } \\
\text { Individual }\end{array}$} & 1.48 & 1.17 & 1.93 & 0.81 & 0.1 & 5.5 \\
\hline & 26.85 & 21.3 & 35.19 & 14.81 & 1.85 & \\
\hline & 5.38 & 5.81 & 5.97 & 5.02 & 2.7 & \\
\hline & 5 & 2 & 4 & 0 & 0 & 11 \\
\hline \multirow{4}{*}{$\begin{array}{l}\text { For-profit - } \\
\text { Limited } \\
\text { Liability }\end{array}$} & 0.25 & 0.1 & 0.2 & 0 & 0 & 0.56 \\
\hline & 45.45 & 18.18 & 36.36 & 0 & 0 & \\
\hline & 0.93 & 0.51 & 0.63 & 0 & 0 & \\
\hline & 106 & 61 & 75 & 29 & 8 & 279 \\
\hline \multirow{4}{*}{$\begin{array}{l}\text { For-profit - } \\
\text { Partnership }\end{array}$} & 5.4 & 3.11 & 3.82 & 1.48 & 0.41 & 14.21 \\
\hline & 37.99 & 21.86 & 26.88 & 10.39 & 2.87 & \\
\hline & 19.67 & 15.4 & 11.79 & 9.09 & 10.81 & \\
\hline & 1 & 0 & 2 & 1 & 2 & 6 \\
\hline \multirow{4}{*}{$\begin{array}{l}\text { Government - } \\
\text { City }\end{array}$} & 0.05 & 0 & 0.1 & 0.05 & 0.1 & 0.31 \\
\hline & 16.67 & 0 & 33.33 & 16.67 & 33.33 & \\
\hline & 0.19 & 0 & 0.31 & 0.31 & 2.7 & \\
\hline & 0 & 1 & 0 & 2 & 0 & 3 \\
\hline \multirow{4}{*}{$\begin{array}{l}\text { Government - } \\
\text { City/county }\end{array}$} & 0 & 0.05 & 0 & 0.1 & 0 & 0.15 \\
\hline & 0 & 33.33 & 0 & 66.67 & 0 & \\
\hline & 0 & 0.25 & 0 & 0.63 & 0 & \\
\hline & 2 & 4 & 6 & 5 & 2 & 19 \\
\hline \multirow{4}{*}{$\begin{array}{l}\text { Government - } \\
\text { County }\end{array}$} & 0.1 & 0.2 & 0.31 & 0.25 & 0.1 & 0.97 \\
\hline & 10.53 & 21.05 & 31.58 & 26.32 & 10.53 & \\
\hline & 0.37 & 1.01 & 0.94 & 1.57 & 2.7 & \\
\hline & 0 & 0 & 1 & 0 & 0 & 1 \\
\hline \multirow{4}{*}{$\begin{array}{l}\text { Government - } \\
\text { Federal }\end{array}$} & 0 & 0 & 0.05 & 0 & 0 & 0.05 \\
\hline & 0 & 0 & 100 & 0 & 0 & \\
\hline & 0 & 0 & 0.16 & 0 & 0 & \\
\hline & 18 & 13 & 16 & 8 & 7 & 62 \\
\hline \multirow{3}{*}{$\begin{array}{l}\text { Government - } \\
\text { Hospital } \\
\text { directed }\end{array}$} & 0.92 & 0.66 & 0.81 & 0.41 & 0.36 & 3.16 \\
\hline & 29.03 & 20.97 & 25.81 & 12.9 & 11.29 & \\
\hline & 3.34 & 3.28 & 2.52 & 2.51 & 9.46 & \\
\hline \multirow{2}{*}{$\begin{array}{l}\text { Government - } \\
\text { State }\end{array}$} & 0 & 1 & 5 & 1 & 1 & 8 \\
\hline & 0 & 0.05 & 0.25 & 0.05 & 0.05 & 0.41 \\
\hline
\end{tabular}

72 Journal of Strategic Innovation and Sustainability Vol. 14(5) 2019 


\begin{tabular}{lrrrrrr} 
& 0 & 12.5 & 62.5 & 12.5 & 12.5 & \\
& 0 & 0.25 & 0.79 & 0.31 & 1.35 & \\
Non profit - & 1 & 8 & 13 & 8 & 5 & 35 \\
Church & 0.05 & 0.41 & 0.66 & 0.41 & 0.25 & 1.78 \\
related & 2.86 & 22.86 & 37.14 & 22.86 & 14.29 & \\
& 0.19 & 2.02 & 2.04 & 2.51 & 6.76 & \\
Nonprofit - & 14 & 22 & 59 & 55 & 16 & 166 \\
Corporation & 0.71 & 1.12 & 3 & 2.8 & 0.81 & 8.45 \\
& 8.43 & 13.25 & 35.54 & 33.13 & 9.64 & \\
& 2.6 & 5.56 & 9.28 & 17.24 & 21.62 & \\
Nonprofit - & 2 & 2 & 0 & 3 & 1 & 8 \\
Other & 0.1 & 0.1 & 0 & 0.15 & 0.05 & 0.41 \\
& 25 & 25 & 0 & 37.5 & 12.5 & \\
Total & 0.37 & 0.51 & 0 & 0.94 & 1.35 & \\
\hline P<0.001 & 539 & 396 & 636 & 319 & 74 & 1964 \\
& 27.44 & 20.16 & 32.38 & 16.24 & 3.77 & 100 \\
\hline
\end{tabular}

\section{CONCLUSIONS}

Nursing homes in the Southwestern United States are spatially located in large metropolitan areas; as seen in maps of nursing homes generated, nursing homes are sparsely located in rural areas, and are often fraught with various measures of low quality. Maps of nursing homes and quality measures from the Nursing Home Compare data illustrated that nursing homes in the Southwestern U.S. are low quality, have high health inspection deficiencies, and are inadequately staffed.

Quality, staffing, and health inspection deficiencies are correlated to ownership status (Structure based measure). Reduced staffing is associated with reduced quality rating in nursing homes, and reduced health inspection ratings. The results of the cluster analysis indicate that overall, nursing homes with high quality are not tightly clustered together.

This study has both methodological strengths and weaknesses. The use of the Nursing Home compare data provided a detailed look at various aspects of quality in Medicare-Medicaid certified nursing home providers. Another strength is that this study also incorporated a large sample size of nursing homes. Data regarding nursing home quality and spatial location was complete and accurate; there were very few missing or unavailable data on nursing home quality. The data confirmed the correlation between quality ratings, ownership, staffing, and health inspection deficiencies, from the literature. In addition, the sample size considered here was large, with 2,037 nursing homes in the southwest United States considered; data was "representative," as data on each and every Medicare-Medicaid certified nursing home provider was analyzed.

On the other hand, the Southwestern states considered in this paper have the highest amount of forprofit nursing home providers ( $>75 \%$ in each state), which may act as an effect modifier for additional quality measures, to be explored; for example, Texas, Louisiana, and Oklahoma have been documented as having the highest percentages of nursing homes with 1 out of 5 star quality ratings, compared to the rest of the United States (Kaiser Family Foundation, 2015). This study may not be generalizable nationwide. Since the locations of nursing homes are dependent on population size and density, the cluster analysis should be interpreted with caution. Another limitation of this study is the use of the ANNI is that the exact numerical value of the quality, staffing, or health inspection ratings was not incorporated within the data analysis. Nursing homes (either 3, 4, or 5), were considered, and the exact magnitude of the rating was not considering in the coefficient/ratio. 
The results of this study should be interpreted in the economic and geographical context of nursing home supply/demand. Geographic differences in the supply of nursing homes, specifically nursing home beds, has been documented (CDC, 2013). These geographic differences in supply of nursing home beds is almost twice as likely to occur in community beds (CDC, 2013). Use of services also varies geographically; for example, in Texas, the use of nursing home services is higher than the national average (CDC, 2013). Population size plays a role in the number of nursing homes in each city and the demand for healthcare services for this population. Research has shown that quality of care varies by the size of the nursing home provider/facility (Kaiser Family Foundation, 2015). There is also geographical variation in the nursing home facility size (Kaiser Family Foundation, 2015).

Future research should also incorporate population density, facility/spatial demographic characteristics, capacity, and payer source. The regional and census tract level demand for nursing homes services should be incorporated into the analysis; state level analyses are not enough. The number of individuals over the age of 65 in each state indicates the number of individuals who can qualify for nursing home services; for example, in Texas there are 3,099,081 residents over the age of 65 (U.S. Census, 2015). Arkansas, Oklahoma, and Louisiana have 466,191, 562,531, and 632,894, respectively, residents over the age of 65 (U.S. Census, 2015). However, this is not representative of the spread of nursing homes/supply for services and the demand for services at the local level. For example, in Arkansas County, Arkansas has 3,250 individuals over the age of 65 as of 2014, while Harris County, Texas has 409,083 individuals over the age of 65, and as mentioned previously, demand for care/usage of services is higher in Texas than in other states (U.S. Census, 2015). In addition, evaluating changes in quality of nursing home care over time would provide a better understanding of resident needs and effective policies.

The significance of this study using GIS software for business information systems and nursing home quality is for governmental accreditation and policy organizations; such healthcare business organizations are thus able to make effective decisions to evaluate changes in nursing home quality in regional locations, using available, secondary data on nursing home quality. In addition, the results of this study are relevant to nursing homes, as the effective use of GIS technology becomes helpful to plan nursing home services to meet demand, especially in rural areas.

\section{REFERENCES}

Castle, N. G. (2008). Nursing home caregiver staffing levels and quality of care a literature review. Journal of Applied Gerontology, 27(4), 375-405. doi:10.1177/0733464808321596

Castle, N. G., \& Ferguson, J. C. (2010). What Is Nursing Home Quality and How Is It Measured? The Gerontologist, 50(4), 426-442. http://doi.org/10.1093/geront/gnq052

Centers for Disease Control and Prevention (CDC). (2013). Retrieved from

https://www.cdc.gov/nchs/data/nsltcp/long_term_care_services_2013.pdf

Centers for Medicare \& Medicaid Services (CMS). (2016). Nursing Home Compare [Homepage]. Retrieved from https://www.medicare.gov/nursinghomecompare/search.html [Data set]

Environmental Science Research Institute (Esri). (n.d.a.). GIS dictionary: Tobler's First Law of Geography. Retrieved from

http://support.esri.com/en/knowledgebase/GISDictionary/term/Tobler's\%20First\%20Law\%20of \%20Geography

Environmental Science Research Institute (Esri). (n.d.b.). Average Nearest Neighbor (Spatial Statistics). Retrieved from http://resources.esri.com/help/9.3/arcgisdesktop/com/gp_toolref/spatial_statistics_tools/average_n earest_neighbor_spatial_statistics_htm

Folland, S., Goodman, A.C., \& Stano, M. (2013). The economics of health and health care. Upper Saddle River, NJ: Pearson Education, Inc. 
Grabowski, D. C., Feng, Z., Hirth, R., Rahman, M., \& Mor, V. (2013). Effect of nursing home ownership on the quality of post-acute care: An instrumental variables approach. Journal of Health Economics, 32(1), 12-21.

Harrington, C., Olney, B., Carrillo, H., \& Kang, T. (2012). Nurse Staffing and Deficiencies in the Largest For-Profit Nursing Home Chains and Chains Owned by Private Equity Companies. Health Services Research, 47(1pt1), 106-128.

Kaiser Family Foundation. (2007). Nursing Home Quality: Twenty years after the Omnibus Reconciliation Budget Act of 1987. Retrieved from https://www.kff.org/wpcontent/uploads/2013/01/7717.pdf

Kaiser Family Foundation. (2015). Reading the Stars: Nursing Home Quality Star Ratings, Nationally and by State. Retrieved from http://kff.org/medicare/issue-brief/reading-the-stars-nursing-homequality-star-ratings-nationally-and-by-state/

Mantravadi, S., \& Zhang, K (2017). Using Geographic Information Systems to Capture Spatial. Patterns in Nursing Home Quality in the Southwestern U.S. 2017 Association for Business Information Systems Conference Proceedings, 2017, 24-32.

Texas A \& M University (TAMU) Geoservices. (2019). Geocoding services [2015]. Retrieved from http://geoservices.tamu.edu/Services/Geocode/

U.S. Census Bureau. (2015). American Fact Finder. Retrieved December 2015 from http://factfinder.census.gov/faces/nav/jsf/pages/index.xhtml

U.S. Census Bureau. (2016). Regions and Divisions. Retrieved from http://www.census.gov/econ/census/help/geography/regions_and_divisions.html

Werner, R. M., \& Konetzka, R. T. (2010). Advancing nursing home quality through quality improvement itself. Health Affairs, 29(1), 81-86. doi:10.1377/hlthaff.2009.0555

Zheng, N. T., Mukamel, D. B., Friedman, B., Caprio, T. V., \& Temkin-Greener, H. (2015). The effect of hospice on hospitalizations of nursing home residents. Journal of the American Medical Directors Association, 16(2), 155-159. http://dx.doi.org/10.1016/j.jamda.2014.08.010 